\title{
Kawasaki disease complicated by subluxation of cervical vertebrae (Grisel syndrome)
}

\begin{abstract}
We describe an unusual presentation of Kawasaki disease (KD) associated with Grisel syndrome (non-traumatic atlantoaxial subluxation most commonly caused by an inflammatory process). While KD with retropharyngeal abnormality is recognised, this is the first reported Australian case of KD associated with atlantoaxial subluxation.
\end{abstract}

Anna J Wood BCom, BA, MB BS

Resident

Davinder

Singh-Grewal MB BS, FRACP, PhD

Paediatric Rheumatologist ${ }^{2}$

Sukanya De

MB BS, FRACP

General Paediatrician ${ }^{3}$

Hasantha Gunasekera

MB BS, FRACP, PhD

General Paediatrician ${ }^{3}$

1Department of Medicine University of Sydney, Sydney, NSW.

2 Department of

Rheumatology,

The Children's Hospital

at Westmead

Sydney, NSW.

3 Department of General

Medicine, The Children's

Hospital at Westmead

Sydney, NSW.

Anna.wood@

austin.org.au

doi: 10.5694/mjal2.11794

\section{Clinical record}

A previously healthy 8-year-old girl presented with a 6-day history of fever and sore throat associated with markedly reduced oral intake and pain and stiffness in her neck. Examination revealed bilateral conjunctival injection, an erythematous tongue and pharynx with enlarged tonsils, a reticular blanching rash over her abdomen, back and thighs, and palmar erythema. She also had bilateral enlarged and tender cervical lymph nodes and torticollis with lateral neck flexion to the left and rotation to the right. Results of the remaining systemic examination, including cardiovascular examination, were normal.

Investigations at presentation included a white blood cell count, which was elevated at $26.7 \times 10^{9} / \mathrm{L}$ (reference interval [RI], $\left.4.5-13 \times 10^{9} / \mathrm{L}\right)$ and indicated neutrophilia (neutrophils, $23.5 \times 10^{9} / \mathrm{L}$ [RI, $\left.1.5-8 \times 10^{9} / \mathrm{L}\right]$ ). The patient's inflammatory markers were elevated; her erythrocyte sedimentation rate was $134 \mathrm{~mm} / \mathrm{h}$ (RI, $<20 \mathrm{~mm} / \mathrm{h}$ ) and Creactive protein level was $344 \mathrm{mg} / \mathrm{L}$ (RI, <10 mg/L). Cerebrospinal fluid examination showed mild pleocytosis, with $11 \times 10^{6} / \mathrm{L}$ mononuclear cells (RI, 0 ), $3 \times 10^{6} / \mathrm{L}$ polymorphonuclear leukocytes (RI, $<5 \times 10^{6} / \mathrm{L}$ ) and $4 \times 10^{6} / \mathrm{L}$ red blood cells (indicative of a traumatic tap; RI, 0). Results of other tests, including serum electrolyte, urea, creatinine, albumin and liver enzyme levels, were normal. Serological tests for Epstein-Barr virus, cytomegalovirus, measles and Streptococcus species were negative, as were blood, cerebrospinal fluid and urine cultures. A neck ultrasound identified multiple enlarged bilateral cervical lymph nodes; the largest was on the left and measured $1.9 \times 1.6 \times 1.2 \mathrm{~cm}$. A lateral neck $x$-ray, although limited by the torticollis, showed a normal retropharyngeal soft tissue space.

A provisional diagnosis of Kawasaki disease (KD) was made, and on Day 8 of her illness the patient was given intravenous immunoglobulin (IVIG, $2 \mathrm{~g} / \mathrm{kg}$ ) with aspirin $(5 \mathrm{mg} / \mathrm{kg} /$ day, which is a common dosage used in Australia). In view of the severe and ongoing neck stiffness, neck pain and sore throat, the possibility of retropharyngeal abscess was also considered. She was therefore given broad-spectrum antibiotic cover (cefotaxime and lincomycin). Her neck pain was managed with regular paracetamol, ibuprofen and oxycodone, and her neck stiffness was

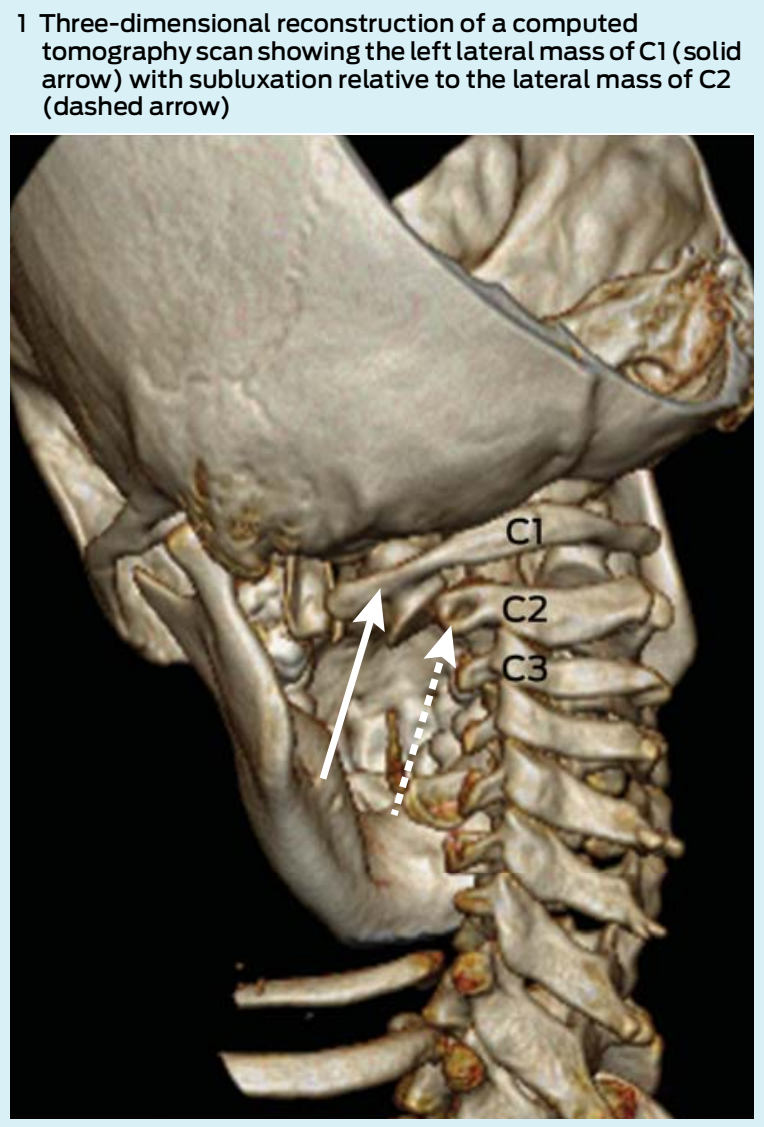

managed with diazepam. Being unable to tolerate solids, she was on a semi-solid diet. Within 48 hours of being given IVIG, the patient's fever abated and the features of KD resolved. An echocardiogram on Day 10 of the illness was normal. She developed thrombocytosis on Day 12, supporting the clinical diagnosis of KD.

On Day 11, in view of ongoing neck pain and torticollis, a computed tomography (CT) scan of the patient's neck showed rotational atlantoaxial subluxation, with $\mathrm{C} 1$ rotated to the right relative to $\mathrm{C} 2$, and persistent bilateral cervical lymphadenopathy (Box 1 and Box 2). The other feature on the CT scan was a hypodense region in the 
2 Axial adjacent slices of a neck computed tomography scan showing $\mathrm{Cl}$ (solid arrow) aligned appropriately with the mandible while C2 (dashed arrow) is rotated
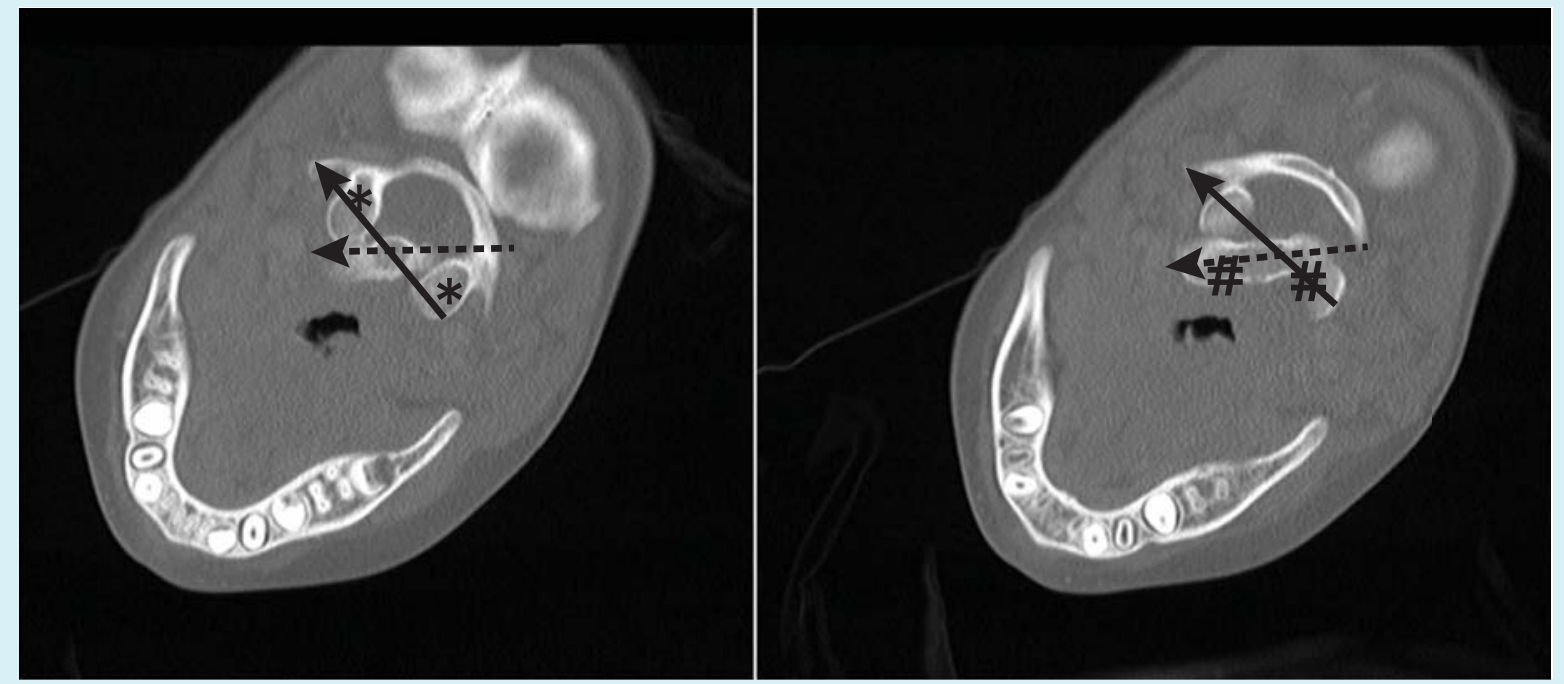

Rotation has resulted in subluxation at the C1-C2 articulation. The lateral masses of C1 (*) do not articulate with the lateral masses of C2 (\#).

retropharyngeal area measuring $6 \mathrm{~mm}$ anteroposteriorly and $7.6 \mathrm{~mm}$ mediolaterally, possibly indicating a retropharyngeal abscess (Box 3). Intravenous cefotaxime and lincomycin were therefore continued for 14 days. The patient was placed in cervical traction for 8 days to reduce the subluxation, followed by visor orthosis (a rigid vest and collar) to immobilise her neck.

A repeat CT scan on Day 22 of the illness showed the atlantoaxial subluxation had resolved. There was mild prevertebral soft tissue oedema but no prevertebral gas or collection. Bilateral cervical lymphadenopathy was still present. The patient was clinically well and was discharged on visor orthosis. She remained well and a 6-week followup CT scan showed normal alignment with no subluxation. The visor orthosis was left in place for 9 weeks, and the patient wore a hard collar for another 2 weeks. Echocardiograms at 4 weeks and 6 months were normal. Regular orthopaedic, cardiac and general medical review was continued.

\section{Discussion}

KD was first described in 1967, but the exact aetiology remains unknown. It is an acute febrile illness associated with a multisystem vasculitis with features including some or all of: bilateral bulbar conjunctival injection with perilimbal sparing; oral mucous membrane changes including injected or fissured lips, injected pharynx and strawberry tongue; cervical lymphadenopathy that is often unilateral; polymorphous rash; and hand and feet changes, including erythema of the palms or soles, oedema of the hands or feet and periungual desquamation. ${ }^{1}$

Non-traumatic atlantoaxial subluxation, known as Grisel syndrome, is an uncommon complication of upper neck inflammation and head and neck surgery. ${ }^{2}$ The first report of KD associated with cervical subluxation, in a patient from Japan, was published recently. ${ }^{3}$ The Japanese case was published after our search of Medline, EMBASE and PubMed with the search strategy Kawasaki disease AND subluxation in March 2012. Ours is the second report, and the first to describe an Australian patient.

Patients with non-traumatic atlantoaxial subluxation present with neck pain and torticollis — with the head tilted towards and the chin rotated away from the side of subluxation (cock-robin deformity) — and, rarely, dysphagia. Results of neurological examination are usually normal. Subluxation begins as torticollis caused by spasm of irritated neck muscles. If longstanding, it may result in relaxation of ligaments and, eventually, subluxation. The exact pathogenesis of subluxation in Grisel syndrome is still debated. ${ }^{4}$

In children, the spinal ligaments are more lax and the atlantoaxial facet joints are shallower and more horizontally orientated than in adults, explaining why subluxation is almost exclusively seen in children. ${ }^{4}$

\section{Neck computed tomography scan showing thickened soft tissue (arrow) anterior to the vertebral bodies, consistent with retropharyngeal inflammation}

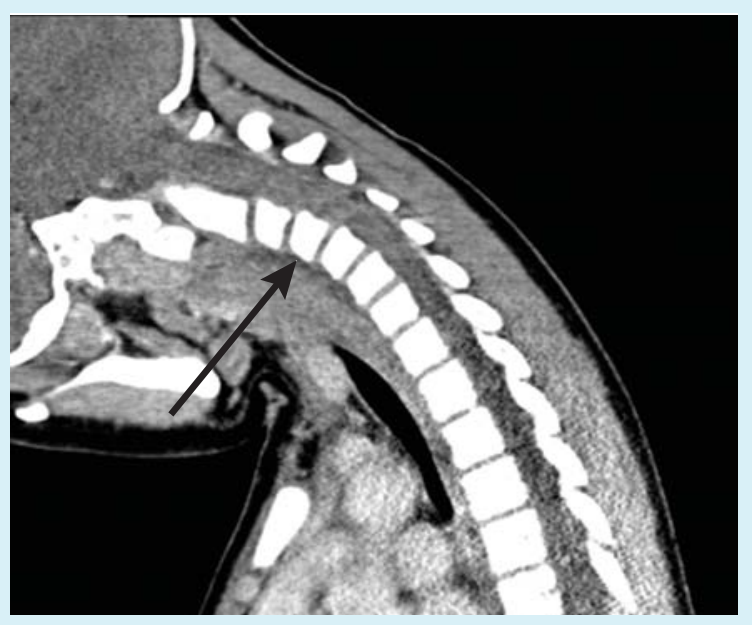


The abnormal head and neck position make plain radiographs difficult to interpret, so a CT scan of the neck is the diagnostic image of choice. The recommended treatment of atlantoaxial subluxation usually involves traction. Less severe forms may respond to conservative treatments such as muscle relaxants and a hard cervical collar, although skull traction is usually necessary to reduce the subluxation. Once reduction is obtained, immobilisation of the cervical spine via orthosis is required for at least 6 weeks. ${ }^{4}$ The length of time before reduction seems to correlate with the likelihood of recurrence and failure of closed reduction techniques. If the subluxation is longstanding, surgical fusion may be required. If left untreated, sequelae can range from mild paraesthesias to quadriplegia to acute respiratory failure. ${ }^{3}$ However, when promptly and effectively treated, the prognosis is excellent.

Lymphadenopathy is the least common diagnostic feature in $\mathrm{KD}$, occurring in $50 \%-75 \%$ of patients; the other four main clinical criteria occur in $90 \%$ of patients. ${ }^{1}$ Patients with KD occasionally present with fever and neck pain alone, making it difficult to differentiate between KD and retropharyngeal infection or abscess. The true incidence of retropharyngeal abnormality in KD is not known, owing to a lack of routine imaging; ${ }^{5}$ however, there are a number of reports of patients with KD who presented with cervical lymphadenopathy and fever, primarily, before the characteristic features of KD became evident. ${ }^{6}$ In most of these cases, intravenous IVIG was administered and the patient's condition improved dramatically. Half of the reported patients with retropharyngeal pathology were diagnosed with KD on or after the tenth day of illness, and most had coronary artery dilatation on echocardiography. In contrast, our patient was diagnosed on Day 8 of the illness, with all five diagnostic criteria in evidence. Despite prompt treatment, she continued to complain of neck pain and severely restricted movements. Further investigation confirmed atlantoaxial subluxation and possible retropharyngeal abscess.

The pathophysiology of the association between KD and retropharyngeal abnormality is unclear. CT scans of the neck in the previously reported cases and in our patient revealed hypodense lesions suggestive of a retropharyngeal abscess. While CT has $90 \%$ sensitivity for detecting deep neck lesions, some studies showing only $60 \%$ specificity for differentiating between inflammation and infection have cast doubt on whether CT scans can identify the cause of the lesion. ${ }^{7}$ It has been suggested that once a patient is diagnosed with KD, the abscess-like lesion on the CT scan should be viewed as a type of inflammation rather than infection. ${ }^{8}$ In our patient, magnetic resonance imaging may have been superior for differentiating between inflammation and infection but would probably have been inferior for assessing for subluxation and would have been technically difficult because of traction equipment.

Furthermore, all but one of the previously reported patients with $\mathrm{KD}$ and possible retropharyngeal abscess improved significantly after treatment with IVIG, and those who underwent exploratory surgery had negative cultures and no abscess. Therefore, it is likely that this CT feature does not represent a true abscess but an inflammatory response. ${ }^{6}$
There is one published case report of KD associated with a pharyngeal abscess requiring incision and drainage. ${ }^{9}$ The 3-year-old boy showed no improvement with antibiotics, and surgical exploration revealed purulent fluid which, when cultured, grew Staphylococcus aureus. It is possible that $S$. aureus had a role as a superantigen in this patient, as concurrent infection has been demonstrated in onethird of patients with a clinical diagnosis of KD. ${ }^{10}$

$\mathrm{KD}$ is not uncommon among patients presenting to major paediatric centres, and the association with retropharyngeal soft tissue inflammation or infection is well documented. We have reported the second case of retropharyngeal inflammation leading to torticollis and atlantoaxial subluxation of cervical vertebrae (Grisel syndrome). Timely detection of atlantoaxial subluxation and appropriate intervention is crucial to achieving a favourable outcome. Clinicians should consider the possibility of subluxation in patients with torticollis as a complication of head and neck infection and inflammation such as KD.

Acknowledgements: We thank Denise Warner, paediatric radiologist, for selecting and describing the appropriate images to show our findings.

Competing interests: No relevant disclosures.

Received 10 Dec 2012, accepted 22 Jun 2013.

1 Ayusawa M, Sonobe T, Uemura S, et al. Revision of diagnostic guidelines for Kawasaki disease (the 5th revised edition). Pediatr Int 2005; 47: 232-234.

2 Wetzel FT, La Rocca H. Grisel's syndrome. Clin Orthop Relat Res 1989; (240): 141-152.

3 Nozaki F, Kusunoki T, Tomoda Y, et al. Grisel syndrome as a complication of Kawasaki disease: a case report and review of the literature. Eur J Pediatr 2013; 172: 119-121.

4 Bredenkamp JK, Maceri DR. Inflammatory torticollis in children. Arch Otolaryngol Head Neck Surg 1990; 116: 310-313.

5 Homicz MR, Carvalho D, Kearns DB, Edmonds J. An atypical presentation of Kawasaki disease resembling a retropharyngeal abscess. Int J Pediatr Otorhinolaryngol 2000; 54: 45-49.

6 Langley EW, Kirse DK, Barnes CE, et al. Retropharyngeal edema: an unusual manifestation of Kawasaki disease. J Emerg Med 2010; 39: 181-185.

7 Weber AL, Siciliano A. CT and MR imaging evaluation of neck infections with clinical correlations. Radiol Clin North Am 2000; 38: 941-968, ix.

8 Hung MC, Wu KG, Hwang B, et al. Kawasaki disease resembling a retropharyngeal abscess - case report and literature review. Int J Cardiol 2007; 115: e94-e96.

9 Choi SH, Kim HJ. A case of Kawasaki disease with coexistence of a parapharyngeal abscess requiring incision and drainage. Korean J Pediatr 2010; 53: 855-858.

10 Benseler SM, McCrindle BW, Silverman ED, et al. Infections and Kawasaki disease: implications for coronary artery outcome. Pediatrics 2005; 116 : e760-e766.

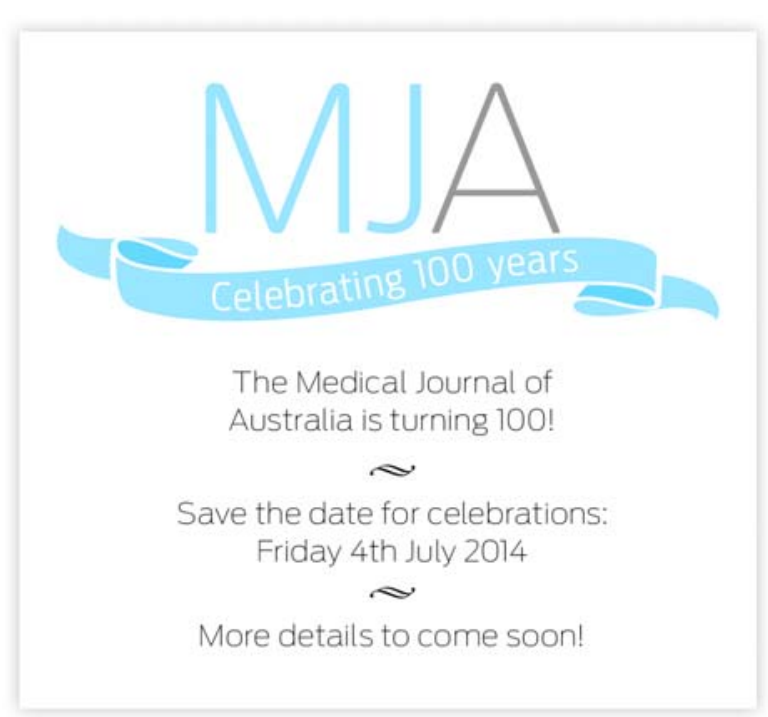

